\title{
The Importance of Nutrition Therapy as a Contributor to the Success of Cancer Treatment
}

\author{
(1) Özlem SÖNMEZ
}

Department of Medical Oncology, Acibadem Mehmetali Aydin University, İstanbul-Turkey

\begin{abstract}
SUMMARY
Cancer is a leading cause of morbidity and mortality in the world. Although developments in early cancer detection and treatments led to improved survival, malnutrition remains to be a significant problem that may affect the response to treatments. Cancer patients remain to be at high risk for malnutrition secondary to local and systemic/metabolic effects of tumors and cancer treatments. The mechanism of cancer cachexia is multifactorial and includes inadequate nutritional intake and systemic inflammation that leads to a metabolic imbalance. Weight loss and cachexia are of prognostic significance and are closely linked to the quality of life. Since early detection and intervention lead to improved outcomes, screening and monitoring nutritional status are critical components of cancer care leading to adequate nutritional therapies. This review article aims to provide an overview of the mechanisms of malnutrition and cancer related cachexia and recent guidelines with the current evidence on the importance of nutrition therapy for cancer patients.
\end{abstract}

Keywords: Cancer; nutrition assessment; nutrition status; malnutrition; sarcopenia.

Copyright $\odot$ 2020, Turkish Society for Radiation Oncology

\section{Introduction}

Cancer is ranked as one of the most important health problems and leading causes of death in the world. Although advances in early detection and treatments contribute to improved survival, many cancer patients continue to experience malnutrition and weight loss as a result of low nutritional intake, emotional distress and metabolic factors related to tumors and treatments. Nutritional status is found to be a strong predictor of cancer prognosis, as well as the quality of life. A study of 8660 cancer patients reported a longer survival for patients with a Body Mass Index (BMI) of $>25$. [1]

It is essential to include nutrition as part of the multidisciplinary approach of cancer management in order to improve the survival and quality of life of cancer patients.[2]

Nutritional assessment and intervention as neces- sary are as crucial as accurate diagnosis and treatment administered that the treatment response rates and survival are lower when there is accompanying malnutrition. Further research on cancer nutrition is required since at least $1 / 3$ of cancer patients are affected by malnutrition.[3]

One of the most common mistakes is not initiating nutrition support before substantial weight loss and a decrease in performance status.

Although the optimal time for nutritional support initiation still lacks data, there is consensus on initiating the nutritional assessment as soon as possible. Treatment of cancer malnutrition has a critical role in the overall outcome, and the delay of nutrition therapy may limit the treatment response. Nutrition risk assessment at the beginning allows for early recognition of malnutrition and treatment when needed.[4] 
Hébuterne et al.[5] reported that malnutrition nay develop in $30-60 \%$ of the patients despite nutritional therapy.Therefore, nutritional screening and appropriate nutrition therapy are a part of multidisciplinary cancer care.[6]

Development of evidence based guidelines will help to increase awareness and the transition of research data to clinical applications, leading to an effective combination of oncological and nutritional therapies through oral, enteral or parenteral routes.[5]

\section{Definition and Mechanisms of Cancer-Associated Malnutrition and Cachexia}

Cachexia is a catabolic state characterized with severe weight loss. Unlike anorexia and classic malnutrition, skeletal muscle mass loss is observed in cachexia, while fat mass is least affected. Quality of life and physical performances of patients with cachexia are affected and the response to nutritional therapy is partial. The incidence of cachexia among cancer patients is approximately $50 \%$, and it is the major cause of mortality in $10-20 \%$ of patients. Literature has shown that nutritional status is an indicator of quality of life in $90 \%$ of patients. Main mechanisms of cancer cachexia involve a combination of several factors, including systemic inflammation, reduced nutrition intake, decreased physical activity, fatigue and metabolic changes secondary to cancer and its treatments.[3]

Reduced intake secondary to treatment and iatrogenic malnutrition are also linked to cachexia. Cachexia is a multifactorial syndrome that develops with the contribution of resistant systemic inflammation and causes muscle loss- with or without fat mass loss- secondary to negative protein and energy balance. Therefore, cachexia is part of a functional progressive impairment, which is usually irreversible with nutritional therapy.[$[7,8]$

Systemic inflammation should also be targeted during anticatabolic therapy. Exercise training should be included to increase physical activity and balance energy with substrate intake. The therapy also should aim to reduce factors that cause impairments secondary to antineoplastic treatment that result in impaired food intake and physical activity. Malnutrition should be suspected when BMI $<20 \mathrm{~kg} / \mathrm{m}^{2}$ for individuals younger than 70 years old and $<22 \mathrm{~kg} / \mathrm{m}^{2}$ for individuals older than 70 years old. Fat-free mass index lower than $15 \mathrm{~kg} / \mathrm{m}^{2}$ for females and $17 \mathrm{~kg} / \mathrm{m}^{2}$ for males is associated with unfavorable prognosis. Therefore, nutritional assessment should be initiated with the cancer diagnosis and repeated at intervals aiming to evaluate food intake, weight and BMI changes.
Systemic inflammation activated by the underlying malignancy is one of the most significant causes of this clinicopathological condition causing malnutrition leading to anorexia and weight loss with muscle and tissue loss.[9]

Weight loss, especially with muscle wasting and inflammation, are the prominent features of cancer cachexia. Symptoms like loss of appetite, fatigue and astenia are closely related with these features. Reduced food intake causes muscle wasting and weight loss. However, this muscle mass loss is usually resistant to standard nutritional therapy and results in progressive function loss.

Proinflammatory cytokines like IL-6, TNF- $\alpha$, IL-1, IFN- $\gamma$ are known to increase secondary to malignancy and are involved in the mechanism leading to cachexia.

Furthermore, IL- $1 \beta$ ve TNF have direct negative effects on the management of food intake through the satiety center, ventromedial nuclei as well as the lateral hypothalamus hunger center, thus resulting in reduced food intake. Lipid mobilizing factor (LMF), proteolysis-inducing factor (PIF) and myostatin are molecules secreted directly from the tumor tissue and they are responsible for the weight loss of cancer cachexia. $[8,10]$

\section{The Importance of Muscle Mass Monitoring and Sar- copenia}

Sarcopenia is a term that can be mistaken for cachexia and weight loss; however, it is a condition of muscle mass and function loss without weight loss. Weight gain can be a part of sarcopenia. It is characterized with low muscle mass despite high BMI. Sarcopenic obesity is linked to increased postoperative complications and extended hospital stays. Obese patients should be screened for sarcopenic obesity and malnutrition.[11]

Extensive fat and extracellular fluid can mask the muscle loss. Sarcopenic obesity can be an indicator of unfavorable prognosis and can be an indicator of postsurgical morbidity.

European Working Group on Sarcopenia in Older People (EWGSOP) recommends gait speed measurement to evaluate muscle strength and performance, and hand dynamometry to assess hand grip strength. Muscle mass measurement is indicated when the gait speed is $\leq 0.8 \mathrm{~m} / \mathrm{s}$, and the patient is diagnosed with sarcopenia when it is low. Hand dynamometry is recommended to further evaluate muscle function when the gait speed is $>0.8 \mathrm{~m} / \mathrm{s}$. Muscle mass measurement is indicated if the test results are $<30 \mathrm{~kg}$ for males and $<20 \mathrm{~kg}$ for females.[12] 
Calf circumference measurement is another anthropometric measurement that can be used for sarcopenia diagnosis and according to the EWGSOP recommendations, a measurement of $\leq 31 \mathrm{~cm}$ is an indicator of sarcopenia.

Malnutrition, cachexia and sarcopenic obesity are linked to low quality of life, extended hospital stays, increased treatment costs and morbidity as well as reduced tolerance to chemotherapy and radiotherapy with lower performance status.

Malnutrition may also develop secondary to chemotherapy, radiotherapy and surgery as a result of reduced caloric intake. Extended hospital stays increase the risk of developing malnutrition. Therefore, patients undergoing active treatments should be monitored closely for malnutrition.[13]

\section{Cachexia Classification and Clinical Outcomes of Early Cachexia Treatment}

Early recognition and management of malnutrition are significant for cancer treatment outcomes. International guidelines considered three stages for a grading cancer-related cachexia, precachexia, cachexia and refractory cachexia.

In precachexia, the clinical signs include anorexia and glucose intolerance with involuntary weight loss of $\leq 5 \%$. Cachexia stage is recognized as more than $5 \%$ loss of stable body weight over a 6 month period or BMI $<20 \mathrm{~kg} / \mathrm{m}^{2}$ or sarcopenia with an ongoing loss of weight of $>2 \%$. The refractory cachexia stage is associated with unresponsiveness to anticancer treatments, low performance status and a life expectancy of $<3$ months.[7]

Resistance to treatment is almost inevitable when nutritional therapy is delayed until the late stages of malnutrition and cachexia. Patients with tumors of the gastrointestinal system, head and neck cancers, lung and liver tumors are among the ones with the highest risk of developing malnutrition. Malnutrition is more likely to develop in elderly patients, as well as patients with advanced stage cancers, when compared to younger patients with early stage cancers.[14]

\section{Assessment of Nutritional Status}

Clinical parameters like tumor location, current treatments, weight loss, and factors that impair food intake and absorption, including nausea, vomiting, diarrhea and constipation, anorexia, astenia, decreased physical activity, pain, depression and socioeconomic factors, should all be considered when assessing the nutritional status.[15]
Daily calorie intake and BMI should be considered when planning nutritional therapy. Weight and nutritional status should be routinely monitored and the nutritional intervention should be adjusted accordingly with the guidance of a dietitian. There are several validated screening tools that allow for fast, cheap and sensitive assessment of the nutritional status.[16]

Medical history, anthropometric measurements, nutrition history, diet intake, biochemical parameters, screening tests are the mainstays of nutritional assessment.

Patient's BMI, thigh and mid upper arm circumference, skin fold measurements are recorded during the initial assessment. Weight assessment should include the weight change, as well as the duration of the change, and the information regarding existing ascites and edema.

Given the unstable metabolic parameters and ongoing inflammatory processes in cancer, blood protein levels are not always reliable; however, despite this ESPEN guidelines recommend to use C-reactive protein (CRP) and serum albumin to estimate the extent of systemic inflammation. [17,18]

There are several tools for nutritional status assessment. Frequently used assessment tools for cancer patients include Patient Generated Subjective Global Assessment (PG-SGA), Malnutrition Screening Tool (MST), Nutritional Risk Screening (NRS-2002), Malnutrition Universal Screening Tool (MUST), Subjective Global Assessment (SGA), Mini Nutritional Assessment (MNA).[19]

PG-SGA questions the amount of oral food intake, weight loss, functional capacity symptoms (existence of nausea, vomiting, loss of appetite, pain, fatigue, odynophagia, early satiety, diarrhea/constipation, mucositis, taste and smell disturbances) are recorded to make a global evaluation of patient status. According to PG-SGA findings, patients scoring 0-1 do not need further intervention but require follow-ups. Patients who scored 2-3 and their caretakers should be educated regarding pharmacological symptom management, they should also receive dietitian counseling. Symptom management should be led by a dietitian when the patients score 4-8. A score of $>9$ is an indicator of a critical situation and these patients should be started on intense nutritional therapy.[19]

MST is a simple screening tool and consists of two questions evaluating the recent unintentional weight loss and malnutrition secondary to loss of appetite. A score of $\geq 2$ indicates a malnutrition risk. [20]

NRS-2002 is used for in-patients and is designed to measure the existing malnutrition as well as disease 
severity. The scoring is between $0-6$, a score of $\geq 3$ is indicative of the need for further assessment for potential nutritional therapy.[21]

MUST, is a screening tool of choice for some specific patient groups. It is used worldwide and is validated for cancer patients.[22]

Although SGA is a more comprehensive tool providing morbidity and mortality estimates for in-patients, it requires a physical examination and more time, as well as trained personnel.[23]

Mini Nutritional Assessment-Short Form (MNA$\mathrm{SF}$ ) is developed for elderly patients by Rubenstein et al. to save time by selecting six questions with the strongest correlation from an 18 question form. It evaluates the malnutrition risk by questioning recent physical activity, reduced food intake, weight loss, BMI, psychological stress, neuropsychological problems and acute illness.[20]

Bioelectrical impedance analysis (BIA) sends an unnoticeable amount of electricity throughout the body and works on the principle that the electric current flows at different rates depending on the tissue composition and it can differentiate between fat and muscle cells using their unique conductivity. It is a practical way to define fat and muscle mass changes in cancer patients during follow-up visits. [24]

Dual-energy X-ray absorptiometry (DXA) and Computerized Tomography can also provide information regarding muscle mass. These imaging modalities can be safely used to evaluate body composition during routine oncology assessments.

\section{Is Nutritional Assessment Time Consuming?}

Although guidelines and literature have pointed out the importance of nutritional assessment on the overall outcome of the cancer treatment, it is common practice to ignore the nutritional assessment given the idea that it will take much time that the clinical routine cannot handle. Nutritional assessment should not be a timeconsuming process, while all evaluations are not required to be done at each visit, simple methods, such as weight change evaluation, biochemical test assessment, an NRS-2002 screening test can take a few minutes, and if we add another few minutes for formulating the nutritional therapy plan, it will still not exceed five minutes for such an efficient treatment modality.

\section{Pharmaconutrients}

Essential amino acids, omega-3 fatty acids and nucleotides have been included in treatments to reduce inflammation while supporting the immune system with the hopes of improving cancer outcomes.[25]

"Immunonutrition" is defined as modulation of the immune system by nutrients and food. It is called "Pharmaconutrition" when nutrients are used in higher than physiological amounts. Immunonutrition aims to increase cellular resilience, the integrity of mucosal barriers and balance the systemic inflammatory responses.[26]

Immunonutrients act like pharmacological agents when consumed in doses higher than daily recommended values and they are called pharmaconutrients. There are ongoing studies on the use of glutamine, omega-3 fatty acids, arginine, vitamins and minerals. Pharmaconutrient is a new era of research with several ongoing studies.[27]

\section{What is Nutrition Therapy?}

Nutrition Therapy for cancer patients is recommended according to the tumor stage, tumor location, grading of malnutrition severity. Depending on the severity, it starts with healthy diet advice and continues as oral enteral nutrition, tube feeding and parenteral nutrition.[28]

If the nutrition advice is not sufficient, and the patient receives $<60 \%$ of the required calories during the last 10 days, nutritional support should be initiated with oral, enteral nutrition. Several products exist on the market with a variety of energy/protein values, concentrations and flavors. The product should be chosen according to the patient's estimated need for calories and protein.

Tube feeding should be initiated if the patient did not respond well to previous nutritional therapy steps, the anatomical location of the tube type and enteral product should be chosen specifically for each patient based on their health status and needs. Tubes include nasogastric, gastrostomy and jejunostomy tubes. Parenteral nutrition is the choice of therapy when enteralnutrition is not possible or adequate.[17,29]

Enteral nutrition products involving L-arginine, RNA nucleotide and omega-3 fatty acids are grouped as immunonutrition products. Their role in enteral nutrition of cancer patients is not clear. There is some data suggesting the benefits of perioperative immunonutrition. It can be recommended for patients undergoing major head and neck and abdominal cancer surgeries, 5-7 days before and after surgery.[30]

\section{Conclusion}

ASPEN and ESPEN guidelines recommend that all cancer patients should be screened for malnutrition risk independent of their BMI and body weight at an 
early stage of their treatment course. Nutrition therapy should be personalized and initiated early. Nutritional assessment includes anorexia, body composition, inflammatory markers, resting energy expenditure and physical activity. Guidelines recommended daily intake of $1.0-1.5 \mathrm{~g} / \mathrm{kg} /$ day protein and $25-30 \mathrm{kcal} / \mathrm{kg} /$ day. Multimodality nutritional therapy aims to increase cancer treatment outcomes by increasing nutritional intake and physical activity while reducing inflammation and hypermetabolic stress.

Peer-review: Externally peer-reviewed.

Conflict of Interest: The authors declare no conflict of interest.

\section{References}

1. Martin L, Senesse P, Gioulbasanis I, Antoun S, Bozzetti F, Deans C, et al. Diagnostic criteria for the classification of cancer-associated weight loss. J Clin Oncol 2015;33(1):90-9.

2. Kagan AR. The multidisciplinary clinic. Int J Radiat Oncol Biol Phys 2005;61:967-8

3. Arends J, Baracos V, Bertz H, Bozzetti F, Calder PC, Deutz NEP, et al. ESPEN expert group recommendations for action against cancer-related malnutrition. Clin Nutr 2017;36(5):1187-96.

4. Attar A, Malka D, Sabaté JM, Bonnetain F, Lecomte T, Aparicio T, et al. Malnutrition is high and underestimated during chemotherapy in gastrointestinal cancer: an AGEO prospective cross-sectional multicenter study. Nutr Cancer 2012;64(4):535-42.

5. Hébuterne X, Lemarié E, Michallet M, de Montreuil CB, Schneider SM, Goldwasser F. Prevalence of malnutrition and current use of nutrition support in patients with cancer. JPEN J Parenter Enteral Nutr 2014;38(2):196-204.

6. Muscaritoli M, Lucia S, Farcomeni A, Lorusso V, Saracino V, Barone C, et al. Prevalence of malnutrition in patients at first medical oncology visit: the PreMiO study. Oncotarget 2017;8(45):79884-96.

7. Fearon K, Strasser F, Anker SD, Bosaeus I, Bruera E, Fainsinger RL, et al. Definition and classification of cancer cachexia: an international consensus. Lancet Oncol 2011;12(5):489-95.

8. Muscaritoli M, Anker SD, Argilés J, Aversa Z, Bauer JM, Biolo G, et al. Consensus definition of sarcopenia, cachexia and pre-cachexia: joint document elaborated by Special Interest Groups (SIG) "cachexia-anorexia in chronic wasting diseases" and "nutrition in geriatrics". Clin Nutr 2010;29(2):154-9.

9. Cederholm T, Bosaeus I, Barazzoni R, Bauer J, Van Gossum A, Klek S, et al. Diagnostic criteria for malnu- trition - An ESPEN Consensus Statement. Clin Nutr 2015;34(3):335-40.

10. Argilés JM, Busquets S, Stemmler B, López-Soriano FJ. Cancer cachexia: understanding the molecular basis. Nat Rev Cancer 2014;14(11):754-62.

11. Tewari N, Awad S, Lobo DN. Regulation of food intake after surgery and the gut brain axis. Curr Opin Clin Nutr Metab Care 2013;16(5):569-75.

12. Cruz-Jentoft AJ, Baeyens JP, Bauer JM, Boirie Y, Cederholm T, Landi F, et al. Sarcopenia: European consensus on definition and diagnosis: Report of the European Working Group on Sarcopenia in Older People. Age Ageing 2010;39(4):412-23.

13. Pressoir M, Desné S, Berchery D, Rossignol G, Poiree B, Meslier M, et al. Prevalence, risk factors and clinical implications of malnutrition in French Comprehensive Cancer Centres. Br J Cancer 2010;102(6):966-71.

14. Freijer K, Tan SS, Koopmanschap MA, Meijers JM, Halfens RJ, Nuijten MJ. The economic costs of disease related malnutrition. Clin Nutr 2013;32(1):136-41.

15. Virizuela JA, Camblor-Álvarez M, Luengo-Pérez LM, Grande E, Álvarez-Hernández J, Sendrós-Madroño MJ, et al. Nutritional support and parenteral nutrition in cancer patients: an expert consensus report. Clin Transl Oncol 2018;20(5):619-29.

16. McLaughlin L. Expert consensus on the nutritional therapy for patients with malignancies. Chin Clin Oncol 2013;2(3):20.

17. Arends J, Bachmann P, Baracos V, Barthelemy N, Bertz $\mathrm{H}$, Bozzetti $\mathrm{F}$, et al. ESPEN guidelines on nutrition in cancer patients. Clin Nutr 2017;36(1):11-48.

18. Hopancı Bıçaklı D. Nutritional Assessement of Oncology Patients. In: Çil T, editor. Nutritional Therapy in End-stage Cancer Patients. 1st ed. Ankara: Türkiye Klinikleri; 2020. p.5-7.

19. Ottery FD. Definition of standardized nutritional assessment and interventional pathways in oncology. Nutrition 1996;12(1 Suppl):S15-9.

20. Ferguson M, Capra S, Bauer J, Banks M. Development of a valid and reliable malnutrition screening tool for adult acute hospital patients. Nutrition 1999;15(6):458-64.

21. Kondrup J, Rasmussen HH, Hamberg O, Stanga Z, Ad Hoc ESPEN Working Group. Nutritional risk screening (NRS 2002): a new method based on an analysis of controlled clinical trials. Clin Nutr 2003;22(3):321-36.

22. Boléo-Tomé C, Monteiro-Grillo I, Camilo M, Ravasco P. Validation of the Malnutrition Universal Screening Tool (MUST) in cancer. Br J Nutr 2012;108(2):343-8.

23. Jensen GL, Compher C, Sullivan DH, Mullin GE. Recognizing malnutrition in adults: definitions and characteristics, screening, assessment, and team approach. JPEN J Parenter Enteral Nutr 2013;37(6):802-7.

24. Gupta D, Lammersfeld CA, Burrows JL, Dahlk SL, 
Vashi PG, Grutsch JF, et al. Bioelectrical impedance phase angle in clinical practice: implications for prognosis in advanced colorectal cancer. Am J Clin Nutr 2004;80(6):1634-8.

25. Dupertuis YM, Meguid MM, Pichard C. Advancing from immunonutrition to a pharmaconutrition: a gigantic challenge. Curr Opin Clin Nutr Metab Care 2009;12(4):398-403.

26. Calder PC. Immunonutrition. BMJ. 2003;327(7407):117-8.

27. Akdeniz N, Kaplan MA. Pharmaconutritional Approaches. In: Çil T, editor. Nutritional Therapy in End-stage Cancer Patients. 1st ed. Ankara: Türkiye Klinikleri; 2020. p.27-34.
28. Karpuzoğlu D, Batar N. Onkolojide tercih edilen beslenme durumu tarama ve değerlendirme yöntemleri. Sağlık ve Yaşam Bilimleri Dergisi 2019;1(1):3746.

29. August DA, Huhmann MB, American Society for Parenteral and Enteral Nutrition (A.S.P.E.N.) Board of Directors. A.S.P.E.N. clinical guidelines: nutrition support therapy during adult anticancer treatment and in hematopoietic cell transplantation. JPEN J Parenter Enteral Nutr 2009;33(5):472-500.

30. Çil T, Kesen O. Enteral Nutritional Treatment in Cancer Patients. In: Çil T, editor. Nutritional Therapy in End-stage Cancer Patients. 1st ed. Ankara: Türkiye Klinikleri; 2020. p.22-26. 